\title{
Recuento y determinación de viabilidad de Giardia spp. y Cryptosporidium spp. en aguas potables y residuales en la cuenca alta del río Bogotá
}

\footnotetext{
Marlén Andrea Alarcón, Milena Beltrán, Martha Liliana Cárdenas, María Claudia Campos

Departamento de Microbiología, Facultad de Ciencias, Pontificia Universidad Javeriana, Bogotá, D.C., Colombia.

Introducción. La transmisión de enfermedades de origen hídrico está relacionada con la contaminación de origen fecal en aguas residuales y potables. Estas enfermedades son causadas por la presencia de bacterias, virus y parásitos, los cuales generan altos porcentajes de morbimortalidad, especialmente, en la población infantil. Se han seleccionado Giardia spp. y Cryptosporidium spp. como organismos indicadores de contaminación de origen parasitario y su análisis es útil para evaluar la calidad del agua y determinar el riesgo sanitario. En Colombia se conoce poco sobre la presencia y la concentración de estos parásitos en aguas, razón por la cual es importante continuar su estudio.

Objetivo. El objetivo de este trabajo fue evaluar la presencia de estos protozoos en cinco estaciones de muestreo de la cuenca alta del río Bogotá y en dos sistemas de potabilización de la misma área.

Materiales y métodos. Las técnicas aplicadas para la determinación y el recuento de las formas quísticas fueron: floculación inorgánica para aguas residuales, filtración para aguas potables y pruebas de colorantes vitales para viabilidad.

Resultados. Se confirmó la presencia de Cryptosporidium spp. en dos de las estaciones del río Bogotá y en las dos potabilizadoras. Giardia spp. se encontró en las dos potabilizadoras pero no en el río Bogotá. La viabilidad fue positiva para Cryptosporidium spp. en una muestra proveniente del río, y negativa para las muestras de agua potable.

Conclusiones. Estos resultados muestran la presencia de protozoos en aguas potables y residuales lo cual puede generar riesgo sanitario para la población de dicha zona.
}

Palabras clave: salud pública, microbiología del agua, Giardia, Cryptosporidium.

Presence and viability of Giardia spp. and Cryptosporidium spp. in drinking water and wastewater in the high basin of Bogotá river

Introduction. Faecal contamination in wastewater and drinking water is linked to the dissemination of water related diseases. The bacteria, virus and parasites present in drinking water are responsible for substantial morbidity and mortality, especially among infants. Giardia spp. and Cryptosporidium spp. were the organisms selected as parasite contamination indicators. Their presence serves as a useful tool for evaluating water quality and determining sanitary risk. At present, in Colombia, concentration and occurrence of these parasites is unknown and an immediate assessment was considered necessary.

Objective. Protozoan presence was determined in five sampling stations in the Bogotá river upper basin and in two drinking water plants near the same area.

Materials and methods. The techniques applied for counting encysted forms consisted of inorganic flocculation for wastewater or filtration for drinking water. Fluorogenic vital dyes tested for viability.

Results. The presence of Cryptosporidium spp. was confirmed in two of the sampled stations and at two of the drinking water plants. Giardia spp. was found at two of the drinking water plants but not at the sampled stations. Viable cysts were found for Cryptosporidium spp. in one of the samples from the Bogotá river, but only inviable exemplars were obtained from the drinking water plants. 
Conclusions. The results revealed protozoan presence in drinking and residual water implying the presence of a potential sanitary hazard.

Key words: public health, water microbiology, Giardia, Cryptosporidium

El crecimiento de la población a nivel mundial y, por consiguiente, el aumento en la demanda del agua para diferentes actividades ha generado el deterioro del recurso hídrico. Una de las fuentes de contaminación más frecuente es la de origen doméstico, la cual se caracteriza por la presencia de altas concentraciones de materia orgánica y microorganismos de origen fecal. Estos contaminantes pueden causar alteraciones en el medio ambiente y riesgo sanitario por la transmisión de enfermedades de origen hídrico (1).

La calidad del agua se ha evaluado tradicionalmente por medio de indicadores de origen bacteriano, viral y parasitario. De estos últimos hacen parte los huevos de helmintos y los quistes de protozoos (2).

Los quistes de Giardia spp. y los ooquistes de Cryptosporidium spp. poseen la característica de permanecer en el ambiente por largos periodos de tiempo bajo condiciones adversas y son resistentes a la mayoría de procesos de desinfección química y tratamiento convencional de aguas (3). Se sabe que las principales formas de transmisión directa de los quistes u ooquistes son la vía fecal-oral y el consumo de agua contaminada, y de forma indirecta, el consumo de alimentos regados con agua sin tratar o el uso de abonos con alto contenido microbiano (4).

El nivel de desarrollo de cada país influye en la aparición de los brotes epidémicos. En los países industrializados esta situación puede ser generada por las posibles fallas en el tratamiento de agua para consumo y a que cada vez es mayor la resistencia de estas formas quísticas a químicos, como el cloro utilizado en la potabilización del agua (5).

Correspondencia:

Claudia Campos, Carrera $7^{a}$ No. 43-82, Bogotá, D.C., Colombia.

Teléfono: 320 8320, extensión 4138; fax: extensión 4021 campos@javeriana.edu.co

Recibido: 20/12/04; aceptado: 09/06/05
Por otra parte, en los países en vía de desarrollo, la situación está dada por la falta de recursos para la construcción y la gestión adecuada de las plantas potabilizadoras y depuradoras y a los bajos niveles de educación (2).

Sumado a esto existen deficiencias en el reporte y seguimiento de la presencia de protozoos en la población y en el agua de consumo.

En algunos países, los esfuerzos se han enfocado hacia la detección de las formas quísticas en cuerpos de agua naturales que son utilizadas como fuente de abastecimiento hídrico $(6,7)$.

En la década de los 80 se publicaron los primeros casos y brotes de enfermedad generados por protozoos. Los países más afectados por giardiasis y criptosporidiosis han sido Estados Unidos, Inglaterra, Escocia y Suecia. La epidemia más importante se reportó en Milwaukee, Wisconsin, donde se infectaron 400.000 personas por el consumo de agua potable contaminada con Cryptosporidium spp. (8).

En Latinoamérica se han adelantado estudios en los que se reportan las concentraciones de estos protozoos en aguas superficiales. Es el caso de Argentina con promedios entre 1 y 16 quistes de Giardia spp. por litro y de 1 a 24 ooquistes de Cryptosporidium spp. por litro $(9,10)$ y de Brasil con 33 a 95 quistes de Giardia spp. por litro y 44 a 100 ooquistes de Cryptosporidium spp. por litro (11). En el caso de aguas residuales, se han encontrado cerca de 2.200 quistes de Giardia spp. por litro y 62 ooquistes de Cryptosporidium spp. por litro en Argentina (10), y de 0 a 680 ooquistes de Cryptosporidium spp. por litro en Brasil (12).

En Colombia, la giardiasis junto con la amibiasis constituyen las dos protozoosis patógenas más revelantes (13). Uno de los estudios epidemiológicos más recientes, llevado a cabo en el departamento del Quindío después del terremoto, muestra altos porcentajes de morbilidad a causa de las malas condiciones de higiene y el consumo de agua de suministro del acueducto. 
El estudio se llevó a cabo en una muestra de 217 niños entre 3 y 13 años de 18 asentamientos temporales, en donde se encontró presencia de quistes de Giardia spp. en el $60,4 \%$ de la población. La principal causa de la enfermedad fue el agua de suministro del acueducto que abastecía 15 asentamientos (65\% de casos) debido a los daños ocasionados por el terremoto al sistema y, en menor proporción (46\% de casos), el agua de suministro de tanques que abastecía solamente tres asentamientos. Una explicación de la menor prevalencia de Giardia en niños que consumían agua de tanques individuales es que hubo en ellos una sedimentación adicional que pudo haber reducido la contaminación del agua con Giardia (14).

Aunque no se encuentran datos de la presencia de protozoos en aguas, es de esperarse que, frente a la ausencia de adecuados sistemas de tratamiento de aguas, las heces de estas personas contaminen las aguas superficiales utilizadas para posterior potabilización y uso agrícola.

En Colombia es común el uso de los ríos como receptores de aguas residuales sin tratar, las cuales se utilizan posteriormente para diferentes actividades de tipo doméstico o agrícola. Tal es el caso del río Bogotá, el cual atraviesa algunas de las zonas más densamente pobladas del país, por lo que el uso de sus aguas es de gran importancia para actividades ganaderas y agrícolas, y como fuente de agua para potabilizar. Buena parte de los municipios localizados en la cuenca del río vierten sus aguas domésticas e industriales sin tratamiento previo, por lo que se pueden considerar como una importante fuente de aguas contaminadas (15). Esta situación es común en la mayoría de los ríos del país, sin que se conozca la concentración de microorganismos patógenos presentes, ni el efecto que sobre ellos pueden causar las barreras naturales y artificiales. De aquí la importancia de poseer herramientas de análisis que permitan evaluar la calidad del agua, la eficiencia de los sistemas de tratamiento y el posible riesgo sanitario para la población.

Aunque se han publicado varios métodos para el análisis de estos protozoos, la mayoría presentan dificultad para su uso debido a los altos costos y a los requerimientos de equipos especializados.
Por esta razón, se han seleccionado dos técnicas de fácil adaptación y más económicas a laboratorios de análisis de aguas. Para el análisis de aguas residuales se empleó la técnica propuesta por Vesey et al. (16) que incluye pasos de floculación, sedimentación y centrifugación, y para aguas, la propuesta por la Agencia de Protección Ambiental de los Estados Unidos (EPA) en 1999 (17), que consiste en pasos sucesivos de filtración, elución, concentración y purificación. En este estudio, la etapa de purificación de los quistes y ooquistes propuesta en el método 1623 mediante separación inmunomagnética, se reemplazó por el uso del gradiente de densidad Percoll-sacarosa, debido a los altos costos y la falta del equipo (18).

Para la determinación de la viabilidad de los quistes de Giardia spp. recuperados a partir de los dos tipos de muestras de agua, se utilizó la metodología propuesta por Thiriat et al. (19) y para los ooquistes de Cryptosporidium spp., el método propuesto por Campbell et al. (20). Las técnicas se basan en la adición de colorantes vitales DAPI (4,6-diamino-2-fenilindol) e IP (yoduro de propidio). Para la detección de Giardia spp. y de Cryptosporidium spp. se empleó el estuche comercial Merifluor ${ }^{\circledR}$ Meridian basado en la marcación con anticuerpos monoclonales y fluoresceína.

El objetivo del presente estudio fue determinar la presencia, la concentración y la viabilidad de quistes de Giardia spp. y de ooquistes de Cryptosporidium spp. en aguas residuales y potables de la cuenca alta del río Bogotá como indicadores de contaminación parasitaria de origen fecal.

\section{Materiales y métodos}

Zona de estudio. Para el análisis de agua residual se recogieron muestras del río Bogotá, en cinco estaciones seleccionadas de acuerdo con una campaña previa de muestreo, en las que se evaluaron parámetros fisicoquímicos, bacterianos y virales (15). El tramo evaluado se encuentra entre los municipios de Villapinzón y Chocontá. El muestreo se realizó entre abril y junio de 2003.

Para el análisis de agua potable se recolectaron muestras de las plantas potabilizadoras de los 
municipios de Villapinzón y Suesca. El muestreo se realizó entre agosto y septiembre de 2003.

Estaciones de muestreo de aguas residuales. Las estaciones de muestreo y su correspondiente georreferenciación expresada en grados y decimales de minuto, se citan a continuación:

- Estación 1: antes del municipio de Villapinzón, al lado de la autopista que de Bogotá conduce a Tunja; latitud norte, $05^{\circ} 13.820^{\prime}$; longitud oeste, 7335.612'; cota, 2.764 msnm.

- Estación 2: en el municipio de Villapinzón, cerca de una casa rural, que vierte sus aguas residuales directamente al río Bogotá; latitud norte, $05^{\circ} 13.041^{\prime}$; longitud oeste, $73^{\circ} 35.858^{\prime}$; cota, 2.764 msnm.

- Estación 3: después del vertido de aguas residuales provenientes del municipio de Villapinzón y del vertido de algunas curtiembres; latitud norte, $05^{\circ} 12.644^{\prime}$; longitud oeste, $73^{\circ} 36.038^{\prime}$; cota, $2.730 \mathrm{msnm}$.

- Estación 4: en el interior de una finca ganadera; en este sitio el río ya ha recibido todos los residuos provenientes de las industrias de curtiembres; latitud norte, $05^{\circ} 10.561^{\prime}$; longitud oeste, $73^{\circ} 38.517^{\prime}$; cota, $2690 \mathrm{msnm}$.
- Estación 5: en el municipio de Chocontá, aguas abajo de la descarga de aguas residuales domésticas tratadas, provenientes de esta población; el municipio de Chocontá cuenta con la primera planta de tratamiento de aguas residuales ubicada sobre la cuenca alta del río Bogotá; latitud norte, $5^{\circ} 08.936$ '; longitud oeste, 7341.345'; cota, 2.661 msnm (figura 1).

Estaciones de muestreo de aguas potables. Para el análisis de agua potable se evaluaron dos plantas potabilizadoras: una en Villapinzón y otra en Suesca; cada planta tiene una fuente diferente de abastecimiento de agua. La planta del municipio de Villapinzón tiene como fuente de abastecimiento una quebrada cercana al cauce del río Bogotá, y la planta del municipio de Suesca se abastece del agua proveniente de un pozo con profundidad de $80 \mathrm{~m}$, ubicado cerca al cauce de dicho río (figura 1).

Los sistemas de potabilización de ambos municipios cuentan con las etapas de aireación, coagulación, sedimentación, filtración y cloración.

Además, se tomó una muestra de agua potable en una casa elegida al azar en cada uno de los municipios evaluados, con el objeto de conocer la calidad del agua una vez ha pasado por los

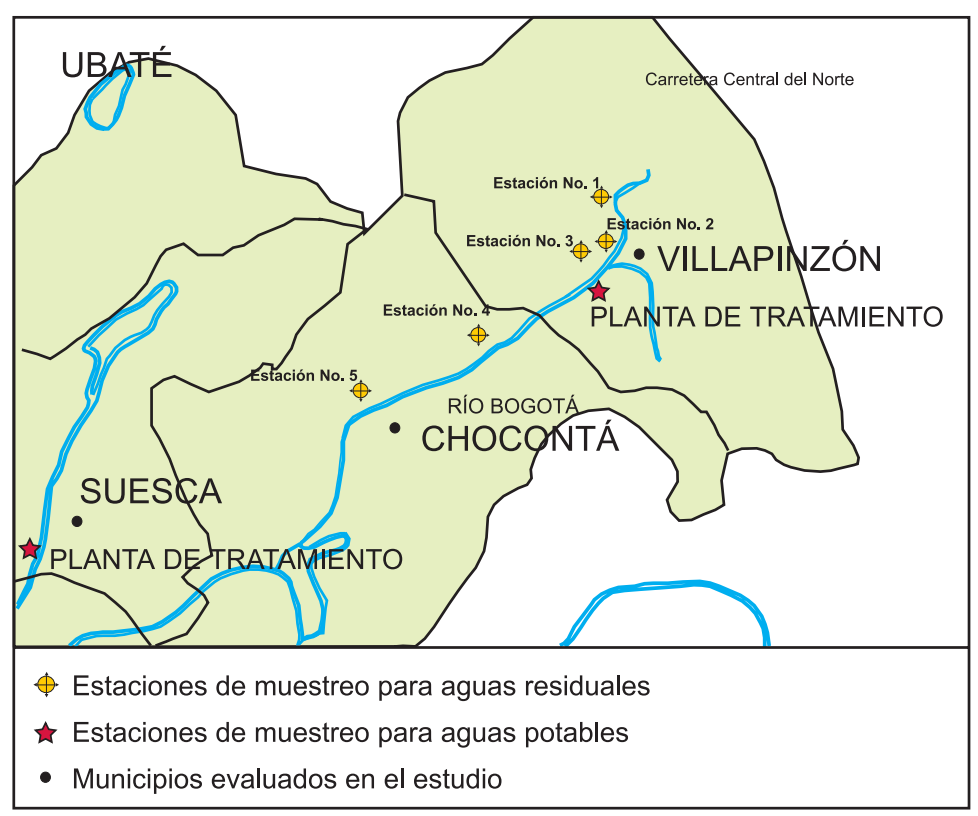

Figura 1. Mapa de georreferenciación de las estaciones de muestreo para aguas residuales y aguas potables pertenecientes a la cuenca alta del río Bogotá. 
sistemas de almacenamiento y distribución. Para el estudio en el municipio de Villapinzón, se eligió una casa ubicada a $2 \mathrm{~km}$, aproximadamente, de la planta de tratamiento y para el estudio en el municipio de Suesca, se escogió la estación de Policía, situada aproximadamente a 1,5 $\mathrm{m}$ de la planta de tratamiento.

\section{Toma de muestras}

Para el análisis de agua residual se recolectaron muestras a partir de las cinco estaciones de muestreo seleccionadas sobre el cauce del río. Se realizaron cuatro muestreos para un total de 20 muestras.

Las muestras se tomaron utilizando un muestreador metálico con un soporte al extremo donde se insertaba un frasco de plástico, el cual se sumergía boca abajo, aproximadamente, $20 \mathrm{~cm}$ por debajo de la superficie del agua a contracorriente y a $3 \mathrm{~m}$ de distancia de la orilla (21).

Los 10 litros de agua recolectados se almacenaron en un garrafón de plástico y se transportaron al laboratorio para ser procesados el mismo día.

Para el análisis de agua potable se recolectaron tres muestras de agua por cada municipio en los siguientes puntos: dos muestras de agua por potabilizadora (una muestra a la entrada y otra a la salida del sistema de tratamiento) y una muestra de agua a partir del grifo de una casa. Estas muestras se recolectaron por día de muestreo para cada municipio, sumando seis muestras de agua potable por día. Se realizaron cuatro muestreos, para un total de 24 muestras.

Las muestras de agua potable se tomaron directamente del tanque de almacenamiento del agua de entrada y de salida de cada una de las plantas potabilizadoras estudiadas. Se utilizaron garrafones de plástico con capacidad de 20 litros. Éstos se sumergieron verticalmente en los tanques con agua y se permitía que se llenaran del líquido hasta alcanzar el volumen necesario para el análisis. Una vez recolectadas las muestras, se transportaban al laboratorio donde se procesaban el mismo día.

En el caso de las muestras de agua provenientes de las casas de los municipios, se abría el grifo y se dejaba correr el agua de 2 a 3 minutos. Luego, se limpiaba con una solución de hipoclorito de sodio (100 mg de $\mathrm{NaOCl}$ por litro) en el orificio de salida del agua y nuevamente se dejaba correr agua otros 2 a 3 minutos. Las muestras de agua se tomaban directamente del grifo (21). Una vez recolectadas en los garrafones, se transportaban al laboratorio para ser procesadas el mismo día.

Teniendo en cuenta el alto costo que requiere el desarrollo de las técnicas propuestas, en especial la técnica para el análisis de agua potable, no se analizó un mayor número de muestras, por lo cual este es un estudio de tipo descriptivo.

Técnica de floculación inorgánica para recuperación y recuento de formas quísticas a partir de agua residual, propuesta por Vesey et al. Las muestras de agua se sometieron a un proceso de floculación mediante la adición de 100 $\mathrm{ml}$ de cloruro de calcio $1 \mathrm{M}$ y $100 \mathrm{ml}$ de bicarbonato de sodio $1 \mathrm{M}$. Luego de agitarlas vigorosamente, se ajustó el pH a 10 con $\mathrm{NaOH} 2 \mathrm{M}$; si la muestra tenía un $\mathrm{pH}$ mayor de 10 no era necesario modificarlo ni corregirlo con ácido. Luego del ajuste del $\mathrm{pH}$, las muestras se dejaron sedimentar por un periodo de 24 horas y se retiró el sobrenadante, recuperándolo en tubos de centrífuga con capacidad de $50 \mathrm{ml}$. Como paso adicional, se lavaron los garrafones con $200 \mathrm{ml}$ de ácido sulfámico al 10\%, $50 \mathrm{ml}$ de PBS, pH 7,4, y $50 \mathrm{ml}$ de Tween 80 al 0,01\% para desprender de las paredes cualquier partícula adherida, y el producto de este lavado también se dispuso en los tubos de centrífuga. El sedimento se centrifugó a $3.000 \mathrm{~g}$ por 10 minutos y se recuperó la mayor cantidad de sedimento en un solo tubo por muestra, haciendo lavados con PBS hasta obtener un $\mathrm{pH}$ final de 7,4. Las muestras concentradas se almacenaron en nevera a $4^{\circ} \mathrm{C}$ hasta su montaje y la lectura (16).

Técnica de filtración para recuperación y detección de Giardia spp. y Cryptosporidium spp. a partir de aguas potables, propuesta por la EPA, método 1623. Las muestras de agua se filtraron a través del filtro Envirocheck $®$ Gelman a una tasa de 2 litros por minuto. Luego, se adicionaron $125 \mathrm{ml}$ de eluyente (mezcla de $10 \mathrm{ml}$ de Laureth 12 al 10\%, $10 \mathrm{ml}$ de Trizma al 12,11\%, $2 \mathrm{ml}$ de EDTA al 18,61\% y $150 \mu \mathrm{l}$ de antifoam A, 
por litro de agua destilada) dentro del filtro y se agitó a 600 rpm por 5 minutos por un solo costado. Este eluido se trasfirió a tubos de centrífuga y se repetió el procedimiento desde la adición del eluyente al filtro hasta la recuperación en los tubos de centrífuga, teniendo en cuenta que antes de la agitación se giraba el filtro $180^{\circ}$ para trabajar por el costado contrario. A continuación, se centrifugó a $1.100 \mathrm{~g}$ por 15 minutos descartando el sobrenadante $1 \mathrm{~cm}$ por encima del sedimento $y$ reuniendo todo el sedimento en un solo tubo. Después de esto, se tomaron $2,5 \mathrm{ml}$ del sedimento con $5 \mathrm{ml}$ de Percoll-sacarosa, densidad=1,1 (45 $\mathrm{ml}$ de Percoll y $10 \mathrm{ml}$ de sacarosa, $2,5 \mathrm{M}$, por 100 $\mathrm{ml}$ de agua destilada) y se centrifugó a $540 \mathrm{~g}$ por 10 minuto, lo cual resultó en la formación de tres fases. La interfase, en la cual se encontraban las formas quísticas, se depositaba en otro tubo de centrífuga y se le lavaron con PBS centrifugando a $2.790 \mathrm{~g}$ por 15 minutos. Las muestras concentradas se almacenaron en nevera a $4^{\circ} \mathrm{C}$ hasta el montaje y la lectura.

Técnica de colorantes vitales con DAPI e IP, propuesta por Campbell et al. y Thiriat et al. Las muestras concentradas a partir de agua residual y potable se sometieron a un procedimiento de marcación con colorantes vitales DAPI (4,6-diamino-2-fenilindol) e IP (yoduro de propidio). Para esto, se mezclaron $100 \mu \mathrm{l}$ de cada muestra con $900 \mu \mathrm{l}$ de reactivo HBSS (tampón de Hank a pH 2,87) durante 1 hora a $37^{\circ} \mathrm{C}$. A continuación, las muestras se centrifugaron y lavaron dos veces con PBS a $14.000 \mathrm{rpm}$ por 30 segundos (17). Se eliminó el sobrenadante y se adicionaron $10 \mu \mathrm{l}$ de DAPI y $10 \mu \mathrm{l}$ de IP en cada tubo, incubando las muestras a $37^{\circ} \mathrm{C}$ durante 2 horas en la oscuridad. Finalmente, se lavaron las muestras con $900 \mu \mathrm{l}$ de PBS, $\mathrm{pH}$ 7,2, centrifugando a $14.000 \mathrm{rpm}$ por 30 segundos.

Marcación con estuche comercial Merifluor ${ }^{\circledR}$ Meridian. De cada muestra marcada con colorantes vitales se tomaron $15 \mu \mathrm{l}$ con asa estéril y se transfirieron a cada uno de los pozos de las láminas del estuche, y se dejaron secar a temperatura ambiente durante 30 minutos (22). Tanto el control positivo como el control negativo del estuche comercial se montaron de la misma manera. El control positivo del estuche contiene una preparación de heces en formalina con ooquistes de Cryptosporidium spp. y quistes de Giardia spp. y el control negativo es una preparación de heces en formalina sin quistes. En condiciones de oscuridad, se agregó una gota del reactivo de detección (preparación de anticuerpos monoclonales anti-Cryptosporidium y anti-Giardia unidos a isocianato de fluoresceína en una solución tamponada que contiene un estabilizante proteico y $0,1 \%$ de azida sódica) y una gota del reactivo de contraste (solución negro de eriocromo) en cada pozo, moviendo circularmente la lámina para permitir la mezcla de los reactivos. Las muestras con los reactivos se incubaron en la oscuridad en cámara húmeda por 30 minutos a temperatura ambiente. El exceso de los reactivos se eliminó con solución tamponada de lavado 20X. Se adicionó una gota de medio de montaje (glicerol tamponado con formalina y $0,05 \%$ de azida sódica) a cada pozo y se cubrieron los pozos con una laminilla, protegiendo las láminas de la luz. Las muestras se almacenaron a $4^{\circ} \mathrm{C}$ en cámara húmeda hasta su lectura.

Lectura. Para la determinación de la viabilidad de las formas quísticas se observó bajo el microscopio de fluorescencia utilizando tres filtros: un filtro con un rango de excitación de 450 a 490 nm que se usó para la detección de quistes u ooquistes teñidos con fluoresceína; un filtro con rango de excitación de 365 a $420 \mathrm{~nm}$ adaptado para observar la coloración con DAPI, y un filtro con un rango de excitación de 520 a 560 nm adaptado para la coloración con IP $(19,20)$. Además, se utilizó el objetivo de contraste de fases para observar la refringencia de las estructuras, confirmando con esta característica la viabilidad de las mismas. Durante la lectura las láminas se mantuvieron en cámara húmeda y en condiciones de oscuridad.

Para el reporte de los resultados se tuvieron en cuenta las características morfológicas de cada protozoo y se empleó el diagrama de criterios de observación establecido por Thiriat et al. para Giardia spp. y lo reportado por Campbell et al. para Cryptosporidium spp.

Para determinar la viabilidad de estas formas quísticas, se deben observar formas ovales con fluorescencia color verde manzana, pared bien 
definida, con tamaño de 8 a $18 \mu \mathrm{m}$ de longitud y 5 a $15 \mu \mathrm{m}$ de ancho que corresponden a Giardia spp. y formas esféricas con fluorescencia color verde manzana, con un diámetro aproximado de 4 a $6 \mu \mathrm{m}$ que corresponden a Cryptosporidium spp. (17); con el colorante DAPI, fluorescencia azul intenso en el quiste completo, dos a cuatro núcleos azul claro en el caso de Giardia spp. y hasta cuatro núcleos azul cielo en el caso de Cryptosporidium spp.; con el colorante IP, ausencia de color para ambas formas quísticas $y$, finalmente, con el objetivo de contraste de fases, los quistes y ooquistes deben verse refringentes. Cualquier característica diferente a las mencionadas anteriormente indica que las formas quísticas no son viables.

La técnica de recuento se realiza observando en el microscopio la totalidad de cada pozo en la lámina, siguiendo un modelo sistemático, de arriba a abajo o de lado a lado (17).

Una vez hecho el recuento se aplica la fórmula que se describe a continuación para obtener el número total de quistes o ooquistes por litro (18):

$$
\mathrm{N}^{\circ} \mathrm{Q} \text { u Ooq } / \mathrm{L}=\underline{\mathrm{N}}^{\circ} \text { contado * Vol. sedimento }
$$$$
\text { Vol. cámara * Vol. muestra }
$$

$N^{\circ}$ contado: formas quísticas viables

Vol. sedimento: volumen del sedimento recuperado Vol. cámara: capacidad del pozo de la cámara (15 $\mu \mathrm{L})$

Vol. muestra: volumen de agua recolectada

Al aplicar la anterior fórmula se reportan los resultados de recuento de protozoos en nuestro estudio. En el caso de no encontrar presencia de protozoos se informó como "no detectado" (ND). El porcentaje de recuperación en aguas residuales para Cryptosporidium spp. fue de $68 \%$ a $79 \%$ (Vesey et al.) y para Giardia spp. entre $57 \%$ y $60 \%$ (comunicación personal: Martha Cárdenas, abril de 2005) y en aguas potables fue de $45 \%$ a $49 \%$ para Giardia spp. y entre $58 \%$ y $62 \%$ para Cryptosporidium spp. (17).

Esta técnica puede presentar interferencias por la turbidez causada por los residuos orgánicos o inorgánicos como arcillas, hierro y polímeros, los cuales pueden interferir en los pasos de concentración, purificación y lectura. Además, algunos organismos como algas, levaduras y otros protozoos pueden dar resultados falsos positivos ya que tienen tamaño similar a los quistes de Giardia spp. y ooquistes de Cryptosporidium spp. y muestran fluorescencia similar a la observada en estos protozoos cuando se colorean con isocianato de fluoresceína $(17,19,23)$.

\section{Resultados}

\section{Muestras de agua residual provenientes de la cuenca alta del río Bogotá}

En el cuadro 1 se observan los resultados de las cinco estaciones de muestreo. Se han incluido los volúmenes empleados para el recuento y el número de quistes y ooquistes encontrados. No se detectó Giardia spp. en los cuatro muestreos realizados para este tipo de agua designados como M1-AR, M2-AR y M3-AR y M4-AR (AR: agua residual). No se detectó Cryptosporidium spp. en los tres primeros muestreos: M1-AR, M2-AR, M3$A R$, pero sí en el último (M4-AR) en las estaciones

Cuadro 1. Resultados del recuento y viabilidad de Giardia spp. y Cryptosporidium spp. en aguas residuales provenientes de la cuenca alta del río Bogotá.

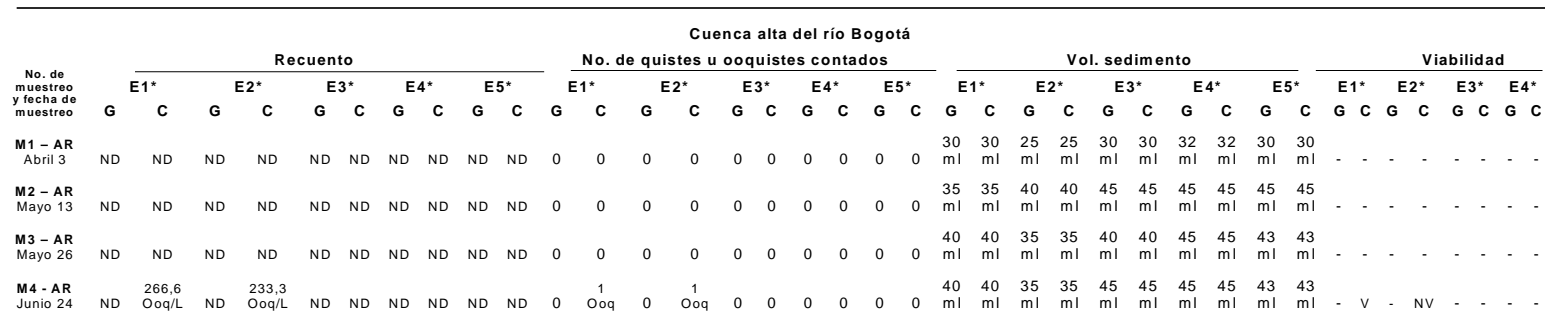

* Estaciones de muestreo establecidas para aguas residuales: E1, estación 1; E2, estación 2; E3, estación 3; E4, estación 4; E5, estación 5: M1-AR: muestreo 1 de agua residual; M2-AR: muestreo 2 de agua residual; M3-AR: muestreo 3 de agua residual; M4-AR: muestreo 4 de agua residual.

G: Giardia spp.; C: Cryptosporidium spp.; Ooq: ooquistes de Cryptosporidium; V: viables; NV: no viables; ND: no detectable; -: por ausencia de protozoos, no se evaluó la viabilidad. 
Cuadro 2. Resultados del recuento y viabilidad de Giardia spp. y Cryptosporidium spp. en aguas potables del municipio de Villapinzón.

\begin{tabular}{|c|c|c|c|c|c|c|c|c|c|c|c|c|c|c|c|c|c|c|c|c|c|c|c|c|}
\hline \multirow{3}{*}{$\begin{array}{l}\text { No. } \\
\text { muestreo } \\
\text { y fecha de } \\
\text { muestreo }\end{array}$} & \multicolumn{6}{|c|}{ Recuento } & \multicolumn{6}{|c|}{$\begin{array}{l}\text { Municipio de Villapinzón } \\
\text { No. de quistes u } \\
\text { ooquistes contados }\end{array}$} & \multicolumn{6}{|c|}{ Vol. sedimento } & \multicolumn{6}{|c|}{ Viabilidad } \\
\hline & \multirow{2}{*}{$\begin{array}{l}\text { En } \\
G\end{array}$} & \multirow{2}{*}{$\begin{array}{c}\text { trada } \\
C\end{array}$} & \multicolumn{2}{|c|}{ a Salida } & \multicolumn{2}{|c|}{ Casa } & \multicolumn{2}{|c|}{ Entrada } & \multicolumn{2}{|c|}{ Salida } & \multicolumn{2}{|c|}{ Casa } & \multicolumn{2}{|c|}{ Entrada } & \multicolumn{2}{|c|}{ Salida } & \multicolumn{2}{|c|}{ Casa } & \multicolumn{2}{|c|}{ Entrada } & \multicolumn{2}{|c|}{ Salida } & \multirow{2}{*}{\multicolumn{2}{|c|}{$\begin{array}{l}\text { Casa } \\
G C C\end{array}$}} \\
\hline & & & $\mathbf{G}$ & C & $\mathbf{G}$ & $C$ & $\mathbf{G}$ & $C$ & $\mathbf{G}$ & $C$ & G & C & $\boldsymbol{G}$ & $C$ & $\mathbf{G}$ & $C$ & $\mathbf{G}$ & $C$ & $\boldsymbol{G}$ & $C$ & & $C$ & & \\
\hline $\begin{array}{l}\text { M1-APV } \\
\text { Agosto } 12\end{array}$ & ND & ND & $\begin{array}{l}10 \\
q / L\end{array}$ & ND & ND & ND & 0 & 0 & $1 q$ & 0 & 0 & 0 & $3 \mathrm{ml}$ & $3 \mathrm{ml}$ & $3 \mathrm{ml}$ & $3 m l$ & $3 m l$ & $3 \mathrm{ml}$ & - & - & NV & - & - & - \\
\hline $\begin{array}{l}\text { M2-APV } \\
\text { Sept. } 1\end{array}$ & $\begin{array}{l}10 \\
q / L\end{array}$ & ND & ND & ND & $\mathrm{ND}$ & ND & $1 q$ & 0 & 0 & 0 & 0 & 0 & $3 \mathrm{ml}$ & $3 \mathrm{ml}$ & $3 \mathrm{ml}$ & $3 \mathrm{ml}$ & $3 \mathrm{ml}$ & $3 \mathrm{ml}$ & NV & - & - & - & - & - \\
\hline $\begin{array}{l}\text { M3-APV } \\
\text { Sept. } 9\end{array}$ & ND & ND 1 & $\begin{array}{c}13,3 \\
q / L\end{array}$ & ND & ND & ND & 0 & 0 & $1 q$ & 0 & 0 & 0 & $3 \mathrm{ml}$ & $3 \mathrm{ml}$ & $4 \mathrm{ml}$ & $4 \mathrm{ml}$ & $4 \mathrm{ml}$ & $4 \mathrm{ml}$ & - & - & NV & - & - & - \\
\hline $\begin{array}{l}\text { M4-APV } \\
\text { Sept. } 23\end{array}$ & ND & ND & ND & $\begin{array}{c}13,3 \\
\text { Ooq/L }\end{array}$ & ND & ND & 0 & 0 & & $100 q$ & 0 & 0 & $3 \mathrm{ml}$ & $3 \mathrm{ml}$ & $4 \mathrm{ml}$ & $4 \mathrm{ml}$ & $4 \mathrm{ml}$ & $4 \mathrm{ml}$ & - & - & -1 & NV & - & - \\
\hline
\end{tabular}

M1-APV: muestreo 1 de agua potable en Villapinzón; M2-APV: muestreo 2 de agua potable en Villapinzón; M3-APV: muestreo 3 de agua potable en Villapinzón; M4-APV: muestreo 4 de agua potable en Villapinzón.

G: Giardia spp.; C: Cryptosporidium spp.; q: quistes de Giardia; Ooq: ooquistes de Cryptosporidium; NV: no viables; ND: no detectable; -: por ausencia de protozoos, no se evaluó la viabilidad.

de muestreo 1 y 2 , con concentraciones de 266,3 y 233,3 ooquistes por litro, respectivamente, y viabilidad positiva únicamente en la estación de muestreo 1. Es importante anotar que en las muestras en las que no se encontró presencia de quistes se informó como ND. No se informa como negativo ya que al tener bajos porcentajes de recuperación (mencionados anteriormente) no se puede descartar del todo la ausencia de los protozoos.

\section{Muestras de agua potable recolectadas en las plantas potabilizadoras ubicadas en la cuenca alta del río Bogotá}

Las muestras recolectadas en las plantas de tratamiento de los municipios de Villapinzón y Suesca fueron positivas para la presencia de Giardia spp. y Cryptosporidium spp. En el cuadro 2 se observa que, en las aguas potables del municipio de Villapinzón, se detectó presencia de Giardia spp. con un recuento de 10 quistes por litro a la salida en el primer muestreo, 10 quistes por litro a la entrada en el segundo muestreo y 13,3 quistes por litro a la salida en el tercer muestreo. Con respecto a Cryptosporidium spp. en esta misma planta, la concentración fue de 13,3 ooquistes viables por litro a la salida en el cuarto muestreo y ND en las muestras restantes. Las formas quísticas encontradas en la potabilizadora de este municipio no presentaron viabilidad. No se detectó ninguno de los protozoos en el agua recogida en la casa.

En el caso de la planta potabilizadora del municipio de Suesca, los resultados evidencian la presencia de ambos protozoos a la entrada de la misma durante el tercer muestreo, con recuentos de 13,3 quistes de Giardia spp. por litro y 10 ooquistes de Cryptosporidium spp. por litro. En los muestreos restantes no se detectaron protozoos. Las formas quísticas encontradas en la planta potabilizadora de este municipio no presentaron viabilidad y en las muestras de agua recogidas en la casa de este municipio no se detectó presencia de ninguno de los protozoos estudiados (cuadro 3).

\section{Discusión}

Evaluación de la presencia y viabilidad de Giardia spp. y Cryptosporidium spp. en la cuenca alta del río Bogotá

La variabilidad en los resultados con relación a la presencia y concentración de estos parásitos puede estar relacionada con la intervención de factores externos como: el efecto de los vertidos 
Cuadro 3. Resultados del recuento y viabilidad de Giardia spp. y Cryptosporidium spp. en aguas potables del municipio de Suesca.

\begin{tabular}{|c|c|c|c|c|c|c|c|c|c|c|c|c|c|c|c|c|c|c|c|c|c|c|c|c|}
\hline \multirow{3}{*}{$\begin{array}{l}\text { No. } \\
\text { muestreo } \\
\text { y fecha de } \\
\text { muestreo }\end{array}$} & \multicolumn{6}{|c|}{ Recuento } & \multicolumn{6}{|c|}{$\begin{array}{l}\text { Municipio de Suesca } \\
\text { No. de quistes u } \\
\text { ooquistes contados }\end{array}$} & \multicolumn{6}{|c|}{ Vol. sedimento } & \multicolumn{6}{|c|}{ Viabilidad } \\
\hline & \multicolumn{2}{|c|}{ Entrada } & \multicolumn{2}{|c|}{ a Salida } & \multicolumn{2}{|c|}{ Casa } & \multicolumn{2}{|c|}{ Entrada } & \multicolumn{2}{|c|}{ Salida } & \multicolumn{2}{|c|}{ Casa } & \multicolumn{2}{|c|}{ Entrada } & \multicolumn{2}{|c|}{ Salida } & \multicolumn{2}{|c|}{ Casa } & \multicolumn{2}{|c|}{ Entrada } & \multicolumn{4}{|c|}{ Salida Casa } \\
\hline & G & $C$ & $\mathbf{G}$ & $C$ & $\mathbf{G}$ & $C$ & G & C & $G$ & $C$ & $\mathbf{G}$ & $C$ & $\boldsymbol{G}$ & $C$ & $\mathbf{G}$ & $C$ & $\mathbf{G}$ & $C$ & G & C & $\mathbf{G}$ & $C$ & G & $C$ \\
\hline $\begin{array}{l}\text { M1-APS } \\
\text { Agosto } 12\end{array}$ & ND & ND & ND & ND & ND & ND & 0 & 0 & 0 & 0 & 0 & 0 & $3 \mathrm{ml}$ & $3 \mathrm{ml}$ & $3 \mathrm{ml}$ & $3 \mathrm{ml}$ & $3 m l$ & $3 \mathrm{ml}$ & - & - & - & - & - & - \\
\hline $\begin{array}{l}\text { M2-APS } \\
\text { Sept. } 1\end{array}$ & ND & ND & ND & ND & ND & ND & 0 & 0 & 0 & 0 & 0 & 0 & $3 \mathrm{ml}$ & $3 \mathrm{ml}$ & $3 \mathrm{ml}$ & $3 \mathrm{ml}$ & $3 \mathrm{ml}$ & $3 \mathrm{ml}$ & - & - & - & - & - & - \\
\hline $\begin{array}{l}\text { M3-APS } \\
\text { Sept. } 9\end{array}$ & $\begin{array}{c}13,3 \\
q / L\end{array}$ & $\begin{array}{c}10 \\
\text { Ooq/L }\end{array}$ & ND & ND & ND & ND & $1 q$ & $100 q$ & 0 & 0 & 0 & 0 & $4 \mathrm{ml}$ & $4 \mathrm{ml}$ & $4 \mathrm{ml}$ & $4 \mathrm{ml}$ & $4 \mathrm{ml}$ & $4 \mathrm{ml}$ & I NV I & NV & - & - & - & - \\
\hline $\begin{array}{l}\text { M4-APS } \\
\text { Sept. } 23\end{array}$ & ND & ND & $\mathrm{ND}$ & ND & ND & $\mathrm{ND}$ & 0 & 0 & 0 & 0 & 0 & 0 & $4 \mathrm{ml}$ & $4 \mathrm{ml}$ & $4 \mathrm{ml}$ & $4 \mathrm{ml}$ & $4 \mathrm{ml}$ & $4 \mathrm{ml}$ & - & - & - & - & - & - \\
\hline
\end{tabular}

M1-APS: muestreo 1 de agua potable en Suesca; M2-APS: muestreo 2 de agua potable en Suesca; M3-APS: muestreo 3 de agua potable en Suesca; M4-APS: muestreo 4 de agua potable en Suesca.

G: Giardia spp.; C: Cryptosporidium spp.; q: quistes de Giardia; Ooq: ooquistes de Cryptosporidium; NV: no viables; ND: no detectable; -: por ausencia de protozoos, no se evaluó la viabilidad.

industriales, la sedimentación de las formas quísticas que no se pueden recuperar a partir de la columna de agua y que se pueden encontrar en el sedimento del río, el bajo número de muestras analizadas en este estudio debido al alto costo de la técnica y a factores físico-químicos del agua como $\mathrm{pH}$, temperatura, turbiedad, sólidos suspendidos y sólidos disueltos, sin olvidar los porcentajes de recuperación de las técnicas (24).

No obstante, en salud pública, además de la cuantificación de los quistes y ooquistes, es muy importante la determinación de la viabilidad de los mismos. En relación a la dosis infecciosa que puede causar enfermedad, se encuentran diferentes datos según la fuente consultada: de 1 a 10 quistes de Giardia spp. y de 1 a 30 ooquistes de Cryptosporidium spp. pueden provocar síntomas clínicos de la enfermedad en humanos $y$, por consiguiente, aumentar el riesgo de infección en la población (1), o de 10 a 25 quistes para Giardia spp. (5) y de 10 a 30 ooquistes para Cryptosporidium spp. (25). Esta determinación no es suficiente por sí sola para confirmar el grado de infectividad y el riesgo que puede provocar en humanos, por lo cual son necesarias otro tipo de pruebas (26). Entre las técnicas usadas para evaluar la infectividad se encuentran las técnicas moleculares que utilizan diversas metodologías basadas en la PCR $(27,28)$ y las biológicas que utilizan la inoculación de animales como herramienta de estudio, ya sea en relación con las concentraciones de protozoos viables que pueden llegar a causar infección en el huésped (29), o con las concentraciones de estas formas quísticas que previamente han sufrido un proceso de inactivación con luz UV y que, posteriormente, se inoculan en animales con el fin de determinar el porcentaje de inactivación de protozoos y la eficiencia de dicha técnica (30).

En relación con los valores encontrados en este trabajo, la concentración de ooquistes de Cryptosporidium spp. en aguas residuales es similar a la reportada por otros autores. Los rangos de valores más frecuentes se encuentran entre $10^{2}$ y $10^{4}$ ooquistes por litro y de $10^{3}$ a $10^{5}$ quistes por litro; los quistes de Giardia spp. son los que se han detectado en mayor concentración, tanto en afluentes como efluentes (31). Sin embargo, los resultados pueden presentar variación dependiendo de los métodos de muestreo, purificación y determinación que se utilicen.

Los estudios realizados en el río Bogotá durante el 2003 y el 2004 (32) para evaluar la calidad microbiológica del agua del río muestran 
concentraciones similares de Cryptosporidium spp. en la cuenca alta, con valores entre 0 y 100 ooquistes por litro. Sin embargo, difiere con respecto a la concentración de Giardia spp., ya que se informan valores entre 0 y 150 quistes por litro. Ninguno de los protozoos presentó viabilidad.

En nuestro estudio, la prevalencia de Cryptosporidium spp. fue mayor a la de Giardia spp., lo cual coincide con los estudios que muestran que los ooquistes de Cryptosporidium spp. son los más resistentes a condiciones ambientales adversas entre las formas quísticas de protozoos (33).

Con relación a la procedencia de los parásitos encontrados, se puede considerar que son de origen fecal humano y animal, ya que de acuerdo al Plan de Ordenamiento Territorial de cada municipio, entre las principales fuentes de contaminación que estarían cercanas a estas las estaciones de muestreo se encuentran: basuras, excretas de origen humano y de animales que se crían en la zona y desechos orgánicos, especialmente, en las zonas rurales de los municipios. En las cabeceras municipales, el mayor aporte de contaminación proviene de vertimientos de aguas residuales domésticas y mataderos sin sistemas de tratamiento.

\section{Evaluación de la presencia y viabilidad de Giardia spp. y Cryptosporidium spp. en aguas potables}

La presencia de estos parásitos puede estar relacionada con la fuente de abastecimiento del agua utilizada para potabilizar, la cual puede presentar algún tipo de contaminación fecal. La segunda causa puede estar relacionada con una falla en el sistema de potabilización del agua teniendo en cuenta que, por lo general, el tratamiento de agua involucra etapas que operan generalmente en serie, las cuales se pueden considerar como puntos críticos de control, por lo que cada etapa debería ser optimizada para producir agua de alta calidad para consumo, independientemente de las condiciones de la fuente $(1,34)$.

En el proceso de coagulación es posible eliminar quistes y ooquistes entre el $90 \%$ y el $95 \%$ (34).
Sin embargo, existen varios factores que se deben controlar en esta etapa, como la dosis adecuada del floculante empleada por volumen de agua de entrada, el tiempo de acción del coagulante y la intensidad de la agitación para garantizar la formación de flóculos que remueven la mayor cantidad de partículas que se encuentran dispersas en el agua (24).

Además, en el proceso de filtración es muy posible que se retengan diferentes partículas sólidas que no sedimentaron en la etapa anterior, entre éstas, los agentes patógenos; de esta forma, se pueden presentar fallas en su eliminación ya sea porque se excede la capacidad de filtración o porque no se limpian adecuadamente los filtros (34).

Por otra parte, en la etapa correspondiente a la cloración del agua, la concentración del cloro aplicada en las plantas potabilizadoras de ambos municipios está alrededor de 0,2 a 1 mg por litro, con un tiempo de contacto mínimo de 30 minutos (2). Sin embargo, esta concentración de cloro es insuficiente para eliminar parásitos y virus, ya que estos microorganismos son resistentes a este desinfectante en dicha concentración. La eliminación puede ser posible empleando mayores concentraciones de cloro y ajustando el tiempo de contacto necesario, según las condiciones de la planta potabilizadora. No obstante, a pesar de obtener mejores resultados, este tipo de práctica no es recomendable ya que se pueden formar compuestos como monocloraminas que pueden generar intoxicaciones y afectar la salud de la población (35). Con relación al proceso de desinfección, el empleo de ozono a una concentración de alrededor de $2 \mathrm{mg} / \mathrm{l}$ es capaz de alcanzar una reducción media en la viabilidad de los ooquistes de $95 \%$ a $96 \%$ con un período de exposición de 10 minutos (36). Sin embargo, la implementación de dicho sistema en los municipios evaluados requiere altos costos de inversión y mantenimiento.

Los resultados encontrados en la planta de potabilización de Suesca parecen indicar que existen mejores condiciones de funcionamiento, ya que no hubo presencia de estas formas quísticas en el agua de salida. Sin embargo, es preciso ampliar el estudio con un mayor número de muestreos. 
No se detectó la presencia de los protozoos en las muestras tomadas directamente del grifo de las casas elegidas en ambos municipios.

Los resultados de este trabajo muestran que los protozoos estudiados, Giardia spp. y Cryptosporidium spp., estuvieron presentes en las aguas residuales provenientes de la cuenca alta del río Bogotá y en los sistemas de potabilización estudiados. Sin embargo, las formas quísticas encontradas no presentaron viabilidad para el caso del agua potable.

Lo anterior pone de manifiesto la presencia de protozoos en la cuenca alta del río Bogotá que, en ocasiones, es utilizada por los pobladores como fuente hídrica para actividades agrícolas y domésticas, lo que puede ser un riesgo potencial de infección en humanos y animales de la zona.

Los resultados obtenidos en el análisis de agua potable revelan deficiencias en la calidad de las fuentes de abastecimiento utilizadas para potabilizar y en el funcionamiento de los sistemas convencionales de potabilización. Si bien los protozoos encontrados a la salida de una de las plantas potabilizadoras no presentan viabilidad, podría indicar que el sistema de potabilización convencional no posee un alto rendimiento en cuanto a la remoción de partículas por sedimentación, ni por filtración del agua de entrada ya que, según los resultados del estudio, se observa que las mismas formas quísticas inactivas encontradas a la entrada de la planta de tratamiento se presentan también a la salida del tratamiento en algunas de las muestras evaluadas. No obstante, estos resultados son similares a los encontrados en estudios en aguas tratadas en Escocia, Inglaterra y Francia, cuyos valores se encuentran entre 10 y $10^{2}$ quistes y ooquistes por litro (31).

En relación con las modificaciones que se le hicieron al método 1623 de la EPA, el empleo de sacarosa, utilizado como gradiente de concentración puede, de algún modo, generar variabilidad en los resultados de viabilidad. Se ha observado que la recuperación con gradientes de sacarosa es poco eficiente, variable y, en cierto grado, afecta la viabilidad de los organismos en la muestra (37). Sin embargo, un estudio más reciente realizado por Stanfield et al. (38) demuestra que el Percoll-sacarosa fue el mejor gradiente de densidad para la recuperación de Giardia spp. En cuanto a los resultados para Cryptosporidium spp., se observaron pequeñas diferencias en comparación con otros gradientes de densidad pero, de igual manera, presentaron un alto porcentaje de recuperación. En la actualidad, se están optimizando los procedimientos que incluyen el uso de separación inmunomagnética y citometría de flujo, debido a que se pueden reducir las posibles pérdidas de organismos en el procesamiento de las muestras (39).

Con relación al paso de filtración utilizado en este estudio, el filtro cápsula Envirocheck ${ }^{\circledR}$ Gelman Laboratory es el sugerido por el método 1623 de la EPA de los Estados Unidos (17). Sin embargo, aunque este filtro en cápsula es el filtro validado en el método 1623, desde hace algún tiempo se trabaja en la validación de otros tres tipos de filtro: el filtro Whatman CrypTest, el filtro cápsula Pall Gelman Envirocheck HV específico para la recuperación de Cryptosporidium y el sistema de filtración IDDEXX Filta-Max (40). Por otra parte, el filtro cápsula Envirocheck permite un alto porcentaje de recuperación de protozoos a partir de bajos volúmenes de agua (41). Al utilizar este tipo de filtro no se presentan daños en las formas quísticas (26).

De otra parte, y según los estudios realizados por Stanfield et al. (38), entre los filtros con mayor porcentaje de recuperación de protozoos en el análisis de aguas potables, se encuentran el Genera Filtra Max, el Cryptoscan (Aldreck) y el Envirocheck ${ }^{\circledR}$ Gelman, este último con porcentajes de recuperación para Giardia del $56,7 \%$ y para Cryptosporidium del $58,1 \%$, evaluado a partir de muestras con concentración de $10^{4}$ quistes u ooquistes por 10 litros.

Este estudio es un paso importante en la aplicación de metodologías para la detección de Giardia spp. y Cryptosporidium spp. en aguas potables y residuales en nuestro medio. Las concentraciones de protozoos encontradas pueden generar riesgo de infección en la población que tiene contacto directo o indirecto con este tipo de aguas, por lo que se sugiere la inclusión de este 
parámetro en la evaluación de la calidad del agua potable en Colombia, tal y como se encuentra en la normativa de varios países.

\section{Agradecimientos}

Agradecemos la colaboración prestada por las empresas de acueducto de los municipios de Villapinzón y Suesca.

\section{Conflicto de interés}

Los autores del presente artículo hacen constar que no tienen ningún tipo de vinculación laboral con los laboratorios que producen los reactivos necesarios para el análisis del recuento y viabilidad de Giardia spp. y Cryptosporidium spp.

\section{Financiación}

Este trabajo fue financiado por la Unión Europea Proyecto No. ERB3514PL972471 de INCO-DC (Internacional Cooperation with Developing Countries) y por la Pontificia Universidad Javeriana.

\section{Referencias}

1. ILSI Argentina, Organización Panamericana de la Salud, Organización Mundial de la Salud. La calidad del agua potable en América Latina. Ponderación de los riesgos microbiológicos contra los riesgos de los subproductos de la desinfección química. En: Gunther FC, Castro R, editores. Washington D.C: Editorial ILSI Press; 1996. p.25-232.

2. Organización Mundial de la Salud. Guías para la calidad del agua potable. 2a. ed. Ginebra: Editorial Alsograf; 1995. p.8-142.

3. Hoogenboezem W, Ketelaars HAM, Medema GJ, Rijs GBJ, Schijven JF. Cryptosporidium and Giardia: occurrence in sewage, manure and surface water. Holanda: Editorial RIWA publishing; 2001. p.24-5.

4. Institute Water Association, World Health Organization, Swedish Institute for Infectious Disease Control. Water Quality: Guidelines, Standards and Health. Assesment of risk and risk management for water-related infectious disease. Ginebra: IWA Publishing; 2001. p.80-306.

5. Hill DR. Giardiasis. Issues in diagnosis and management. Infect Dis Clin North Am 1993;7:503-25.

6. Slifko TR, Couiliette A, Huffman DE, Rose JB. Impact of purification procedures on the viability and infectivity of Cryptosporidium parvum oocysts. Water Sci Technol 2000;41:23-9.

7. Oda T, Sakagami M, Ito H, Yano H, Rai SK, Kawabata $M$ et al. Size selective continuous flow filtration method for detection of Cryptosporidium and Giardia. Water Res 2000;34:4477-81.

8. Mac Kenzie WR, Hoxie NJ, Proctor ME, Gradus MS, Blair KA, Peterson DE et al. A massive outbreak in Milwaukee of Cryptosporidium infection transmitted through the public water supply. New Eng J Med 1994; 331:160-7.

9. Abramovich BL, Gilli MI, Haye MA, Carrera E, Lura MC, Nepote A et al. Cryptosporidium and Giardia in surface water. Rev Argent Microbiol 2001;33:167-76.

10. Calderón E. Evaluating of the usefulness of bacteriophages as model microorganisms for the assessment of water treatrement processes and cooperation with developing countries. INCO DC: International Cooperation with Developing Countries. Buenos Aires: Universidad de Buenos Aires; 2002.

11. Franco RM, Rocha-Eberhardt R, Cantusio Neto R. Occurrence of Cryptosporidium oocysts and Giardia cysts in raw water from the Atibaia river, Campinas, Brazil. Rev Inst Med Trop Sao Paulo 2001;43:109-11.

12. Coutinho EW, Gamba RC, Pellizari VH. Detection of Cryptosporidium spp. oocysts in raw sewage and creek water in the city of Sâo Paulo, Brazil. Brazilian Journal of Microbiology 2002;33:41-3.

13. Corredor A, Arciniegas E, Hernández CA, editores. Parasitismo Intestinal. Bogotá: Instituto Nacional de Salud: 2000. p.9-73.

14. Lora-Suárez F, Marin-Vásquez C, Loango N, Gallego M, Torres E, González MM et al. Giardiasis in children living in post-earthquake camps from Armenia (Colombia). BMC Public Health 2002; 2:5. Disponible en: http://www.biomedcentral.com/14712458/2/5.

15. Campos C, Guerrero A. Evaluating the usefulness of bacteriophages as model microorganisms for the assessment of water treatment processes and water quality final report. INCO-DC. International Cooperation with Developing Countries. Bogotá: Universidad Javeriana; 2002.

16. Vesey G, Slade JS, Byrne M, Shepherd K, Fricker CR. A new method for the concentration of Cryptosporidium oocysts from water. J Appl Bacteriol 1993;75:82-6.

17. U.S. Environmental Protection Agency. USEPA Method 1623: Cryptosporidium and Giardia in water by Filtration/IMS/FA. EPA-821-R-99-006. Office of Water, U. S. Washington D.C; Environmental Protection Agency: 1999.

18. Dellundi J. Puesta a punto de metodologías para la detección de los protozoos Giardia y Cryptosporidium en aguas (tesis). Barcelona: Universitat de Barcelona; 2002.

19. Thiriat L, Sidaner F, Schwartzbrod J. Determination of Giardia cyst viability in environmental and faecal 
samples by immunofluorescence, fluorogenic dye staining and differential interference contrast microscopy. Lett Appl Microbiol 1998;26:237-42.

20. Campbell AT, Robertson LJ, Smith HV. Viability of Cryptosporidium parvum oocysts: correlation of in vitro excystation with inclusion or exclusion of fluorogenic vital dyes. Appl Environ Microbiol 1992;58:3488-93.

21. American Public Health Association, American Water Works Association, Water Environmental Federation. Standard methods for the examination of water and wastewater. 20a. ed. Washington: Editorial United Book Press, Inc; 1998. Secciones: 9-18 y 9-21.

22. Merifluor ${ }^{\circledR}$ Meridian. Inserto del estuche comercial Meriflour ${ }^{\circledR}$ Cryptosporidium/Giardia(Merifluor ${ }^{\circledR}$ C/G) Procedimiento de detección por Inmunofluorescencía Directa de Cryptosporidium y Giardia. Referencia No. 250050.

23. Rodgers MR, Flanigan DJ, Jakubowski W. Identification of algae which interfere with the detection of Giardia cysts and Cryptosporidium oocysts and a method for alleviating this interference. Appl Environ Microbiol 1995;61:3759-63.

24. DeZuane J. Handbook of drinking water quality. 2a. ed. New York: Editorial John Wiley and Sons, Inc; 1997. p.49-320.

25. Okhuysen PC, Chappell CL, Crabb JH, Sterling CR, DuPont HL. Virulence of three distinct Cryptosporidium parvum isolates for healthy adults. J Infect Dis 1999; 180:1275-81.

26. Guyot K, Gireaudot-Liepmann MF, Cabon A, Riveau-Ricard I, Lange M, Delattre JM et al. Influence of US EPA 1622 method successive steps on the viability of Cryptosporidium oocysts. Water Sci Technol 2000;41:189-96.

27. Quintero-Betancourt W, Gennaccaro AL, Scott TM, Rose JB. Assessment of methods for detection of infectious Cryptosporidium oocysts and Giardia cysts in reclaimed effluents. Appl Environ Microbiol 2003; 69: 5380-8.

28. Mayer CL, Palmer CJ. Evaluation of PCR, nested PCR, and fluorescent antibodies for detection of Giardia and Cryptosporidium species in wastewater. Appl Environ Microbiol 1996;62:2081-5.

29. Mehlhorn H, Pierkaski G. Fundamentos de parasitología. 3a. ed. Zaragoza: Editorial Acribia S.A; 1993. p.6-68.

30. Mofidi AA, Meyer EA, Wallis PM, Chou Cl, Meyer BP, Ramalingam $\mathbf{S}$ et al. The effect of UV light on the inactivation of Giardia lamblia and Giardia muris cysts as determined by animal infectivity. Water Res 2002; 36:2098-108.
31. Carraro E, Fea E, Salva S, Gilli G. Impact of a wastewater treatment plant on Cryptosporidium oocysts and Giardia cysts occurring in a surface water. Water Sci Technol 2000;41:31-7.

32. Departamento de Ingeniería Civil y Departamento de Microbiología. Informe final del proyecto "Monitoreo y diagnóstico de la calidad del agua del río Bogotá y su impacto en la salud de las comunidades". Bogotá: Universidad Javeriana; 2004.

33. World Health Organization. Guidelines for drinking water quality. Health criteria and other supporting information. 2a. ed. Geneva: World Health Organization; 1998. p.29-140.

34. Gray NF. Calidad del agua potable. Problemas y soluciones. Zaragoza: Editorial Acribia S.A; 1996. p.49258.

35. Corona-Vásquez B, Rennecker LJ, Driedger MA, Mariñas JB. Sequential inactivation of Cryptosporidium parvum oocysts with chlorine dioxide followed by free chlorine or monochloramine. Water Res 2002;36:17888.

36. Hirata T, Chikuma D, Shimura A, Hashimoto A, Motoyama N, Takahashi K et al. Effects of ozonitation and chlorination on viability and infectivity of Cryptosporidium parvum oocysts. Water Sci Technol 2000;41:39-46.

37. Smith HV, Hayes CR. The status of UK methods for the detection of Cryptosporidium spp. oocysts and Giardia spp. cysts in water concentrates. Water Sci Technol 1997;35:369-76.

38. Stanfield G, Carrington E, Albinet F, Compagnon B, Dumoutier N, Hambsch B et al. An optimized and standardized test to determine the presence of the protozooa Cryptosporidium and Giardia in water. Water Sci Technol 2000;41:103-10.

39. Swales C, Wright S. Evaluation of a continuous flow centrifuge for recovery of Cryptosporidium oocysts from large volume water samples. Water Res 2000; 34:1962-6.

40. McCuin RM, Clancy JL. Modifications to United States Environmental Protection Agency methods 1622 and 1623 for detection of Cryptosporidium oocysts and Giardia cysts in water. Appl Environ Microbiol 2003; 69:267-74.

41. Pezzana A, Vilagines PH, Bordet F, Coquard D, Sarrette B, VIlaginés $\mathbf{R}$. Optimization of the Envirocheck capsule method and inmunomagnetic separation procedure for the detection of levels of Cryptosporidium in large drinking water samples. Water Sci Technol 2000; 41:111-7. 